\title{
The psychological impact of COVID-19 outbreak on medical staff and the general public
}

\author{
Biao Chen ${ }^{1} \cdot$ Qing-xian $\mathrm{Li}^{1} \cdot$ Heng Zhang ${ }^{2}$ • Jia-yong Zhu ${ }^{1} \cdot \mathrm{Xu} \mathrm{Yang}{ }^{1} \cdot$ Yu-hang $\mathrm{Wu}^{3} \cdot$ Jie Xiong ${ }^{4} \cdot \mathrm{Fu} \mathrm{Li}^{4} \cdot \mathrm{Hua} \mathrm{Wang}^{1}$. \\ Zhi-tao Chen ${ }^{2}$ iD
}

Accepted: 2 October 2020 / Published online: 7 October 2020

(C) Springer Science+Business Media, LLC, part of Springer Nature 2020

\begin{abstract}
To assess the psychological effects of the novel coronavirus disease (COVID-19) on medical staff and the general public. During the outbreak of COVID-19, an internet-based questionnaire included The Self-rating Depression Scale (SDS), Perceived Stress Scale (PSS-10), and Impact of Event Scale-Revised (IES-R) was used to assess the impact of the pandemic situation on the mental health of medical staff and general population in Wuhan and its surrounding areas. Among the 1493 questionnaires completed, $827(55.39 \%)$ of these were men, and $422(28.27 \%)$ of these were medical personnel. The results suggest that the outbreak of COVID-19 has affected individuals significantly, the degree of which is related to age, sex, occupation and mental illness. There was a significant difference in PSS-10 and IES-R scores between the medical staff and the general population. The medical staff showed higher PSS-10 scores $(16.813 \pm 4.87)$ and IES-R scores $(22.40 \pm 12.12)$ compared to members of the general population PSS-10 $(14.80 \pm 5.60)$ and IES-R scores $(17.89 \pm 13.08)$. However, there was no statistically significant difference between the SDS scores of medical staff $(44.52 \pm 12.36)$ and the general public $(43.08 \pm 11.42)$. In terms of the need for psychological assistance, $50.97 \%$ of interviewees responded that they needed psychological counseling, of which medical staff accounted for $65.87 \%$ and non-medical staff accounted for $45.10 \%$. During the ongoing COVID-19 outbreak, great attention should be paid to the mental health of the population, especially medical staff, and measures such as psychological intervention should be actively carried out for reducing the psychosocial effects.
\end{abstract}

Keywords Psychological effects · COVID-19 · Outbreak · Medical staff

\section{Introduction}

In December of 2019, a new acute respiratory disease, the novel coronavirus disease (COVID-19), outbreak was firstly

Biao Chen, Qing-xian Li, Heng Zhang, Jia-yong Zhu and Xu Yang contributed equally to this work.

Hua Wang

whzkwlh@126.com

Zhi-tao Chen

chenzhitao830201@163.com

1 Department of Orthopedic Surgery, Zhongnan Hospital of Wuhan University, Wuhan 430071, China

2 Department of Gastroenterology, The Central Hospital of Wuhan, Tongji Medical College, Huazhong University of Science and Technology, Wuhan 430014, China

3 Applied Statistics, School of mathematics and statistics, Wuhan University, Wuhan 430072, China

4 The Mental Health Center of Wuhan, Wuhan 430022, China identified in Wuhan, Hubei Province, China (Huang et al. 2020). Subsequently, the disease spread rapidly across the globe, posing a great challenge to worldwide health care systems (Bogoch et al. 2020; Holshue et al. 2020; Wu et al. 2020). COVID-19 is a highly infectious disease with rapid transmission and short incubation period (Huang et al. 2020; Li et al. 2020). Many patients do not have typical clinical manifestations. Because the disease is mainly transmitted by close contact and respiratory droplets and easily transfers between individuals in the family and hospital settings, the health care workers and the patients' family members are high-risk groups (Riou and Althaus 2020; Chan et al. 2020). As of February 11, 2020, China has confirmed 1716 cases of COVID-19 among medical personnel, accounting for 3.8\% percent of all confirmed cases in the country, according to the State Council press release. Wuhan has 1102 confirmed cases among health care workers, accounting for $73.8 \%$ percent of health care workers in China. Worryingly, so far COVID-19 has become a pandemic and the number of infected population has soared to more than 30 million in the range of the world according to the data presented by the World Health Organization (WHO). 
A recent psychological survey of the Spanish population showed that in the period of COVID-19, $18.7 \%$ of the population showed depressive symptoms, while $21.6 \%$ and $15.8 \%$ developed anxiety and PTSD symptoms respectively, and that gender and with certain diseases which have been previous diagnosed may aggravate psychological symptoms (Gonzalez-Sanguino et al. 2020). The other report showed that in the early stages of the COVID-19 outbreak in China, the mental health status of more than half of the respondents was very seriously affected (Wang et al. 2020a). It shows that the psychological impact of the epidemic on people is huge. Medical staff have played a vital role in this fight against the epidemic. However, the fight against COVID-19 pneumonia is a serious challenge for medical staff, especially for those who work on the front line (Heymann and Shindo 2020). Hard work, lack of sleep, and shortage of medical staff make the challenges of dealing with the infection highly stressful (Chen et al. 2020). It is likely that these combined factors may lead to increases in mental strain, depression, and physical and mental fatigue of medical workers. During the treatment and nursing of COVID-19 patients, some adverse psychological reactions such as fear, depression, and social psychological disorder appeared. For the general population, the lack of understanding of the disease and strict prevention and control measures on the impact of life have exacerbated their psychological stress (Duan and Zhu 2020). If these adverse psychological reactions are unable to be efficiently regulated and solved in time, the degrees of punitive physical and psychological harm may be difficult to estimate.

The Public Health Emergency of International Concern (PHEIC) recently called for an extraordinary public health response, similar to what was observed for SARS, H1N1, Zaire Ebolavirus, and Zika (Qiu et al. 2018; Pfefferbaum et al. 2012; Mbala-Kingebeni et al. 2019; Metsky et al. 2017). However, the COVID-19 outbreak targeted a large number of locations during a short period of time, resulting in increasing concerns about the speed and mode of transmission of the disease, particularly taking into consideration the large number of medical staff and the general population infected in Wuhan (Nishiura et al. 2020). This situation has generated a significant psychological and emotional burden among the anti-COVID-19 medical staff and the general population, which poses a great challenge to government and healthcare systems worldwide (Heymann and Shindo 2020; Duan and Zhu 2020). This report administered questionnaires and surveys to medical staff and members of the general population in order to explore the effects of COVID-19 on mental health. The findings of this report provide a reference for incorporating humanistic care and psychological intervention into the rescue strategies and emergency response procedures to major public health events.

\section{Methods}

The study was approved by the Wuhan Central Hospital Ethics Committee. In order to reduce the potential spread of the virus during direct contact, the survey was administered by using the network questionnaire. All samples were taken randomly from the population living in Wuhan, China, which was the worst epidemic areasand and its surrounding areas from 23 January to 22 March 2020. We divided the population into medical staff group and non-medical staff group according to different occupations. A total of 1600 questionnaires were distributed and received 1493 questionnaires. The questionnaire, consisting of 160 multiple-choice questions and short answer questions, took about $20 \mathrm{~min}$ to complete.

The Self-rating Depression Scale (SDS), Perceived Stress Scale (PSS-10) and Impact of Event Scale-Revised (IES-R) scales were used to evaluate the impact of the epidemic on the mental health of medical staff and the general population during the epidemic period of COVID-19. Each item of SDS was divided into four grades according to the frequency of symptoms: no or little time, little time, considerable time, most or all time. The critical value of depression was 53, in which higher scores indicated greater depressive tendencies (Zung and Wonnacott 1970). The PSS-10 stress scale is designed to measure the perceived subjective stress, and includes six negative description items and four positive feeling description items (Berlinberg et al. 2019). Each item is scored on a 15 scale: never, almost never, sometimes, often, always. A total score of 13 represents a normal stress level, but scores of 20 or higher represent high stress levels which require therapeutic intervention (Katsarou et al. 2012).The IES-R event impact scale is a self-reported measure designed to assess the current subjective distress caused by traumatic life events, consisting of 22 items, each with a $0-4$ Likert rating scale, with the highest score being 88 (Garrouste-Orgeas et al. 2019). The mean IES-R score of 20 or higher considered to be at higher risk for posttraumatic stress disorder (PTSD) (Feinstein et al. 2002).

\section{Statistical Analysis}

Categorical variables are described in terms of frequency and percentage, and continuous variables are described in terms of mean and standard deviation. Using the demographic information of interviewees as the independent variable, we conducted linear regression analyses on the variables SDS, PSS10 , and IES-R to determine whether the demographic variables explained variance in the scale outcomes. The Wilcoxon rank sum test and chi-square test were used to analyze the psychological test results of medical and nonmedical staff to determine differences between the groups. All statistical analyses were performed using SPSS (24.0) and Matlab (R2019a). GraphPad Prism (v8.0) was used to 
create the artwork. In the above tests, $P \leq 0.05$ was considered to be statistically significant.

\section{Results}

\section{Study Population Characteristics}

A total of 1600 questionnaires were distributed and received 1493 questionnaires yielded valid data in this survey. Demographic information was presented in Table 1.

\section{The Relationship between Basic Information and Psychological Changes}

To determine the relationship between demographic characteristics and scores on the IES-R, PSS-10 and related factors of the SDS score, we conducted linear regression analyses. Specifically, we examined the demographic variables of sex, age, education, history of mental illness, the presence of suspected or confirmed COVID-19 cases, occupation, whether the interviewee is still working during the epidemic, and whether the interviewee participated in the frontline response. The regression coefficients were shown in Table 2.

The relationship between related factors and the three scales was shown in Fig. 1. In brief, gender and history of mental illness were positively correlated to IES-R, PSS-10 and SDS scores. There was a negative correlation between IES-R, PSS-10 and SDS scores in the presence of closing to COVID-19 patients or suspected patients. Age was positively correlated with IES-R and PSS-10, but negatively correlated with SDS. Education had no effect on IES-R score, which was positively correlated with PSS-10 score, while negatively
Table 1 Baseline characteristics of interviewees

\begin{tabular}{|c|c|}
\hline Characteristics & $\mathrm{N}(\%)(n=1493)$ \\
\hline \multicolumn{2}{|l|}{ Age(y) } \\
\hline$<18$ & $13(0.87)$ \\
\hline $18-25$ & $572(38.31)$ \\
\hline $26-35$ & $467(31.28)$ \\
\hline $36-45$ & $296(19.83)$ \\
\hline $46-55$ & $119(7.97)$ \\
\hline $56-65$ & $25(1.67)$ \\
\hline$>66$ & $1(0.07)$ \\
\hline \multicolumn{2}{|l|}{ Gender } \\
\hline Male & $827(55.39)$ \\
\hline Female & $666(44.61)$ \\
\hline \multicolumn{2}{|l|}{ Education } \\
\hline Junior middle school or below & $41(2.75)$ \\
\hline Technical secondary school/High school/Vocational high school & $288(19.29)$ \\
\hline Junior college & $841(56.33)$ \\
\hline Master's/Doctor's degree & $323(21.63)$ \\
\hline \multicolumn{2}{|l|}{ Occupation } \\
\hline Medical staff & $422(28.27)$ \\
\hline No-medical staff & 1071(71.73) \\
\hline \multicolumn{2}{|l|}{ If there are any suspected or confirmed COVID-19 cases } \\
\hline Yes & $327(21.90)$ \\
\hline No & $1166(78.10)$ \\
\hline \multicolumn{2}{|l|}{ History of mental illness } \\
\hline Yes & $8(0.54)$ \\
\hline No & 1485(99.46) \\
\hline \multicolumn{2}{|l|}{ Still working during the epidemic } \\
\hline Yes & $866(58.00)$ \\
\hline No & $627(42.00)$ \\
\hline \multicolumn{2}{|l|}{ Participate in the frontline response } \\
\hline Yes & $301(20.16)$ \\
\hline No & $1192(79.84)$ \\
\hline
\end{tabular}


Table 2 Linear regression analysis of baseline characteristics and questionnaire scores

\begin{tabular}{|c|c|c|c|}
\hline & \multicolumn{3}{|c|}{ Regression coefficients, $\mathrm{B}(\mathrm{t})$} \\
\hline & IES-R & PSS-10 & SDS \\
\hline Sex & $4.35\left(6.39^{* *}\right)$ & $0.76\left(2.57^{*}\right)$ & $3.18\left(4.98^{* *}\right)$ \\
\hline Age & $1.56\left(4.71^{* *}\right)$ & $0.98\left(6.82^{* *}\right)$ & $-1.03\left(-3.31^{* *}\right)$ \\
\hline Education & $0.79(1.64)$ & $1.18\left(5.65^{* *}\right)$ & $-1.93\left(-4.25^{* *}\right)$ \\
\hline History of mental illness & $17.36\left(3.88^{* *}\right)$ & $4.38\left(2.26^{*}\right)$ & $18.96\left(4.52^{* *}\right)$ \\
\hline If there are any suspected or confirmed COVID-19 cases & $-4.28\left(-4.61^{* *}\right)$ & $-1.38\left(-3.42^{* *}\right)$ & $-4.38\left(-5.04^{* *}\right)$ \\
\hline Occupation & $-0.04(-0.54)$ & $-0.04(-1.07)$ & $0.02(0.31)$ \\
\hline Still working during epidemic & $3.36\left(4.51^{* *}\right)$ & $0.49(1.53)$ & $2.36\left(3.38^{* * *}\right)$ \\
\hline Participate in the front-line response & $-4.15\left(-4.32^{* *}\right)$ & $-0.33(-0.80)$ & $-2.95\left(-3.27^{* *}\right)$ \\
\hline $\mathrm{R}^{2}$ & 0.19 & 0.15 & 0.13 \\
\hline F & $13.54^{* *}$ & $10.04^{* *}$ & $10.04^{* *}$ \\
\hline
\end{tabular}

${ }^{*} P<0.05,{ }^{* *} P<0.01$

correlated with SDS score. Working during the epidemic showed a positive correlation between IES-R and SDS score, and a statistical difference with PSS-10 score. There was a negative correlation between participate in the frontline response. However, there was no statistical difference on PSS10. Other factors showed no statistical difference in the scores of IES-R, PSS-10, and SDS.

\section{Differences between Questionnaire Scores of Medical Staff and the General Public}

The Wilcoxon rank sum test was conducted in order to determine difference in psychological scores between medical staff and members of the general public. There was no significant difference in SDS score between
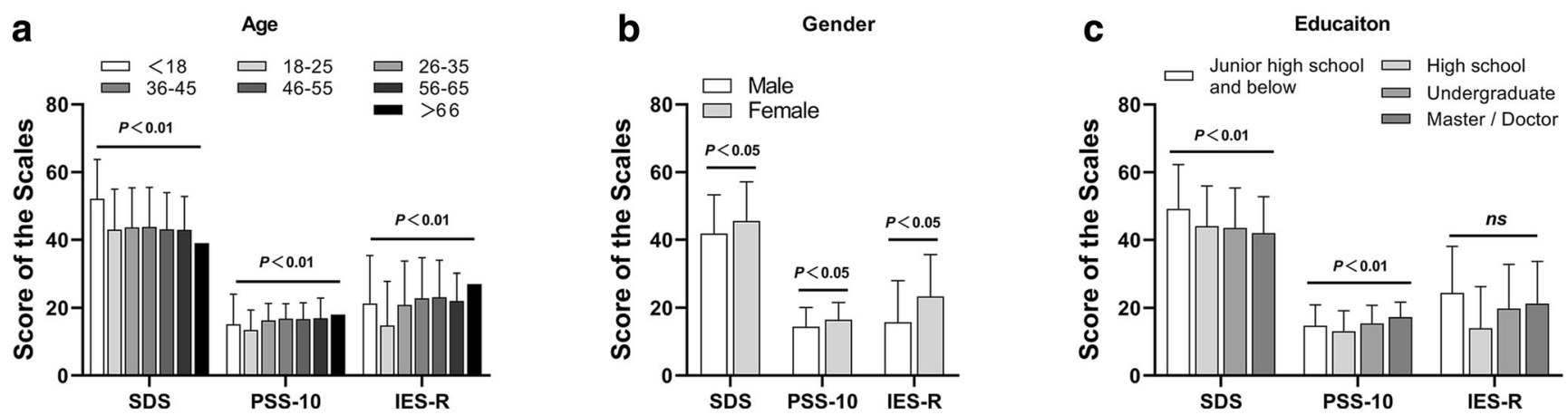

d History of mental illness

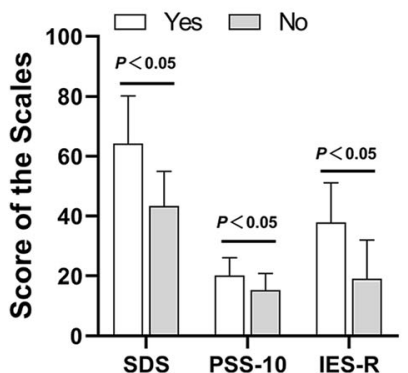

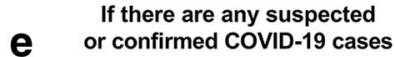

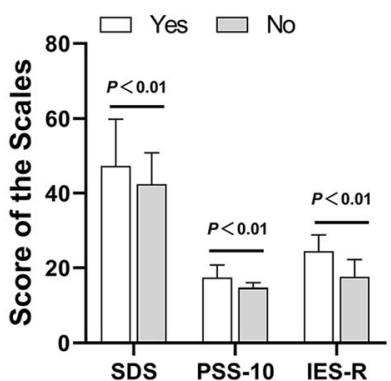

Fig. 1 Relationship between baseline characteristics and psychological changes. Relationship between scores on the three scales [the Self-rating Depression Scale (SDS), the Perceived Stress Scale (PSS10) and the Impact of Event Scale-Revised (IES-R) scales] and the demographic variables of sex (a), age (b), education (c), history of mental
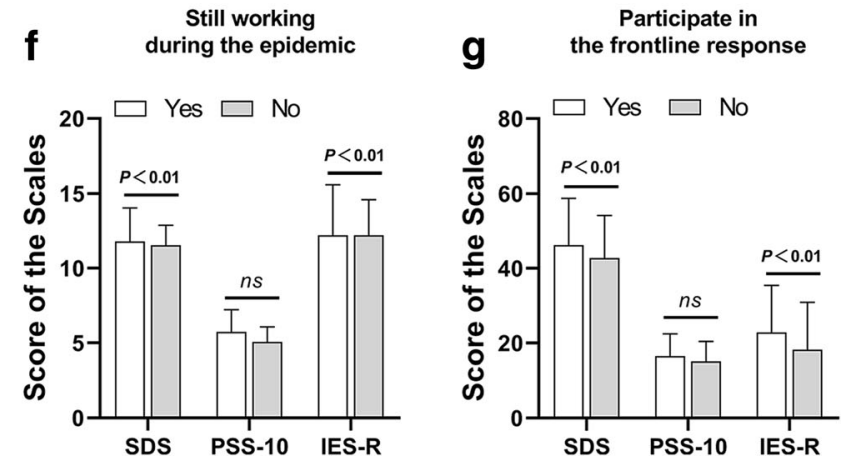

illness (d). the presence of suspected or confirmed COVID-19 cases (e), whether the interviewee is still working during the epidemic (f), and whether the interviewee participated in the frontline response $(\mathbf{g}) . P$ values indicate differences between groups. $P<0.05$ was considered statistically significant 
medical staff $44.52(12.36)$ and the general public 43.08(11.42), $P=0.08$. The PSS-10 score of medical staff 16.81(4.87) was higher than that of general public 14.80(5.60), $P<0.01$. Meanwhile, the IES-R score of medical staff $22.40(12.12)$ was also higher than that of general public 17.89(13.08), $P<0.01$. The results were presented in Table 3.

\section{Differences between Psychological States of Medical Staff and the General Public}

The Chi-square sum test was conducted in order to determine difference in psychological states between medical staff and members of the general public. The SDS score of depression was 53, in which higher scores indicated greater depressive tendencies. There was no significant difference in depressive tendencies between medical staff $(3.79 \%)$ and the general public $(2.89 \%), P=0.47$. The PSS-10 scores of 20 or higher represent high stress levels which require therapeutic intervention. There were more medical staff $(93.36 \%)$ who was in a high stress levels than that of general public $(86.65 \%)$, $P<0.01$. Meanwhile, the IES-R score of 20 or higher considered to be at higher risk for PTSD. There were more medical staff $(71.56 \%)$ who was in a higher risk of PTSD than that of general public $(55.84 \%), P<0.01$. The results were presented in Table 4.

\section{Attitudes and Needs of Medical Staff and the General Public towards Psychological Counseling during the Epidemic}

Of the 1493 respondents, only 428 (28.67\%) had access to psychological counseling and $761(50.97 \%)$ needed psychological counseling. The relative risk ratio (RR) was defined as 1. In this study, it was found that medical staff (40 [9.47\%]) had access to psychological counseling only 0.27 (95\% CI 0.23 to $0.31 ; P<0.01)$ times that of the general public $(388$ [36.23\%]), while the demand for psychological counseling of medical staff (278 [65.87\%]) was 1.46 (95\%CI 1.33 to 1.61; $P<0.01)$ times that of the general public (483 [45.10\%]). The results were presented in Table 5.

\section{Discussion}

The COVID-19 epidemic was initially reported in December 2019 in Wuhan, Hubei Province, China, and it spread to many countries and regions around the world with a staggering rate. To date, there have been many research reports on the basic and clinical treatment of COVID-19 cases (Ai et al. 2020; Wang et al. 2020b; Xu et al. 2020). Studies have shown that at the beginning of the outbreak in Wuhan, medical staff experienced more serious psychological problems (Kang et al. 2020a; Kang et al. 2020b) . However, it is not clear whether the psychological problems of medical staff are milder or more serious compared with non-medical staff. It is therefore extremely important to fully understand the mental health of these populations, especially in the face of public health emergencies such as COVID-19. To this end, we conducted a series of mental health surveys for medical staff and the members of the general public.

In this study, by analyzing mental health scores of the population during the epidemic, we found a significantly high level of psychological stress in the general public, which was more particularly prominent among women and those with mental illness. It is worth noting that there were only 8 respondents with a history of mental illness in this survey, so a larger sample size is needed to further study the impact of COVID-19 on this group of people. Similar to our conclusions, several pieces of research have also shown that the additional psychological stress of COVID-19 may aggravate existing symptoms in patients (especially women) with mental illness, which may subsequently develop more serious consequences such as decreased immunity and potentially increase the incidence of underlying diseases, thereby increasing the risk of being infected by SARS-COV-2 (Gonzalez-Sanguino et al. 2020; Wang et al. 2020a; Stefana et al. 2020; Fischer et al. 2020). We also found that older adults were more likely to be affected, and effects were particularly prominent in nonmedical personnel. The reason for this may be related to the comprehensive understanding of the epidemic situation, the public information of the society, and the ability of people to discern the truth (The 2020). This also explains some seemingly unexpected results. For example, we observed that people with suspected or diagnosed cases around them and those who participated in front-line work during the epidemic
Table 3 Wilcoxon rank sum test results for questionnaire score differences between medical staff and the general public

\begin{tabular}{llll}
\hline & Medical staff, mean (SEM) & General public, mean (SEM) & $P$ values \\
\hline SDS & $44.52(12.36)$ & $43.08(11.42)$ & .08 \\
PSS-10 & $16.81(4.87)$ & $14.80(5.60)$ & $<.01^{* * *}$ \\
IES-R & $22.40(12.12)$ & $17.89(13.08)$ & $<.01^{* * *}$ \\
\hline
\end{tabular}

SDS Self-rating Depression Scale, PSS-10 Perceived Stress Scale, IES-R Impact of Event Scale-Revised scales. ${ }^{*} P<0.05,{ }^{* *} P<0.01$ 
Table 4 Chi-square test results for psychological states differences between medical staff and the general public

\begin{tabular}{llll}
\hline & & Medical staff & General public \\
\hline SDS, N(\%) & greater depressive tendency & $16(3.79)$ & $31(2.89)$ \\
& no depressive tendency & $406(96.21)$ & $1040(97.11)$ \\
& $\chi^{2}$ & 0.53 & \\
& $P$ values & 0.47 & \\
PSS-10, N(\%) & high stress levels & $394(93.36)$ & $928(86.65)$ \\
& normal stress level & $28(6.64)$ & $143(13.35)$ \\
& $\chi^{2}$ & 12.81 & \\
IES-R, N(\%) & $P$-values & $<.01^{* *}$ & $598(55.84)$ \\
& posttraumatic stress disorder & $302(71.56)$ & $473(44.16)$ \\
& non-posttraumatic stress disorder & $120(28.44)$ & \\
& $\chi^{2}$ & 36.63 & \\
& $P$-values & $<.01^{* *}$ & \\
& & &
\end{tabular}

SDS Self-rating Depression Scale, $P S S-10$ Perceived Stress Scale, IES-R Impact of Event Scale-Revised scales. ${ }^{*} P<0.05,{ }^{* *} P<0.01$ seemed to be better mental health, and these results were found mainly in medical staff. This result may be due to the fact that they were still active on the front-line, and their tense working state made them temporarily not paying attention to changes in their physical and psychological conditions and even prompted the psychological defense, and the fatigue caused by adverse psychological effects and mental symptoms may appear later. However, the psychological health of the general population who were still working during the epidemic was worse. This may be due to the increased risk of infection and excessive fatigue in the pandemic that caused a certain degree of panic among many non-medical staff. In general, our results suggested that the large-scale outbreak of COVID-19 affected individuals on a wide scale, the degree of which was impacted by age, sex, occupation, mental illness, and employment status.

In examining the mental status of medical and non-medical staff using three different scales of mental health, we observed that the epidemic appeared to have resulted in overwhelmingly negative attitude towards many events in life. Compared to the non-medical staff, vast majority of medical staff participating in the front-line epidemic prevention showed significantly higher PSS-10 scores. This phenomenon may reflect the responsibilities of medical staff and psychological stress of close contact with patients. On the other hand, it may also be that the majority of medical personnel with heavy protective gear working in isolation wards face a large degree of psychological shock from the overwhelming information emerging about the disease, which may exacerbate feelings of pessimism and anxiety about the event.

Following the outbreak of COVID-19, it was found through epidemiological investigations that the virus had higher infectivity and a longer incubation period compared to SARS (Mahase 2020). These remarkable characteristics brought the entire community a huge psychological impact. The results of IES-R showed that an extended period of exposure to negative information related to the outbreak caused a considerable number of medical and non-medical staff to experience what might be similar to a post-traumatic stress response. The main manifestations of this were sleep disorders, anxiety caused by the mention of the epidemic, oversensitivity and unconscious emergence to the relevant information of the epidemic. Traumatic stress came from a variety of factors, including the severity of the epidemic, developmental trends, duration, and control measures. In combination with the current status of the epidemic, we showed that people were fearful when they realized an epidemic was happening around them.

Table 5 Comparison of the needs of medical staff and the general public for psychological counseling

\begin{tabular}{|c|c|c|c|c|c|}
\hline & $N(\%)$ & Medical staff $(n=422),(\%)$ & General public $(n=1071),(\%)$ & $\mathrm{RR}(95 \% \mathrm{CI})$ & $P$-value \\
\hline Access to psychological counseling ${ }^{a}$ & $428(28.67)$ & $40(9.47)$ & $388(36.23)$ & $0.27(0.23 \sim 0.31)$ & $<.01^{* * *}$ \\
\hline Demand for psychological counseling & $761(50.97)$ & $278(65.87)$ & $483(45.10)$ & $1.46(1.33 \sim 1.61)$ & $<.01^{* * *}$ \\
\hline
\end{tabular}

${ }^{a}$ Psychological counseling: including psychological consultation, psychotherapy, psychological examination or psychological tests, etc.

${ }^{*} P<0.05,{ }^{* *} P<0.01$ 
The results of the SDS depression scale showed no significant differences in scores between medical and non-medical staff, although the impact of the incident caused by the epidemic resulted in some symptoms of depression. For medical staff, they were at the center of pathogen exposure and were very susceptible to virus infection, could place a strain on psychological endurance. At the same time, they stayed away from loved ones for long periods of time, leading to observable depressive symptoms. The possibility of medical personnel becoming infected might negatively impact the confidence of the general public, and factors such as the increasing number of confirmed diagnoses and deaths and enforced isolation might lead decreasing mental health.

For medical staff, negative emotions were not conducive to their mental health status and might negatively affect the treatment of patients. For non-medical staff, especially patients, poor mental health might increase the risk of various diseases, such as digestive, endocrine, nervous system, and immune system diseases, thereby increasing the risk of viral infection by COVID-19 (Pariante 2016; Adam et al. 2017; Park and Jeen 2016). Therefore, effective psychological counseling and psychological intervention are necessary. From our results, it can be seen that since the outbreak, $28.67 \%$ of the interviewees received psychological counseling, of which 9.47\% were medical personnel and $36.23 \%$ were nonmedical personnel. In addition, $50.97 \%$ responded that they needed psychological counseling, of which medical staff accounted for $65.87 \%$ and non-medical staff accounted for $45.10 \%$. These results suggested that of the considerable number of individuals who expressed need psychological assistance, medical staff accounted for the majority. We must therefore attach great importance to the mental health of medical workers and actively provide psychological counseling and psychological support to improve the overall quality of mental health in medical staff (Yang et al. 2020). At the same time, society also ensure that psychological care is provided to the general public, including adequate health education to improve people's awareness of the disease and reduce public fears. Given our results, women and older adults should be the focus of attention, and they should be receiving widespread attention from mental health workers, hospital managers, and society.

There are some limitations in this study. First, the questionnaire survey time is selected for one month. Follow-up studies on the longer-term mental health effects of population need to be further explored. Secondly, the sample size of the questionnaire survey is limited, a larger samples survey can further reduce the bias. In addition, there is a certain gap between the sample size of medical staff and non-medical staff, and the sanitary conditions of the working environment of medical staff are obviously not as good as non-medical staff (home isolation), so these two factors may have a certain impact on the results.

\section{Conclusion}

Our results suggested that during the epidemic, both medical staff and non-medical staff experienced psychological stress, especially the psychological problems of medical staff were more serious. In the wake of the COVID-19 outbreak, there has been widespread attention to the basic research and clinical treatment of the disease. However, equal attention should be also be paid to the mental health status of medical staff working in environments where the disease is present. Psychological consultation and support should be actively carried out in order to improve the overall mental health of medical staff. The results of this study also suggest that measures such as humanistic care and psychological intervention should be included in the rescue strategies and plans for emergency responses to major public health incidents in order to reduce the consequences of infection to the general population.

Data Availability Statement All data generated or analysed during this study are included in this published article.

Funding This research was supported by the Zhongnan Hospital of Wuhan University Science, Technology and Innovation Seed Fund (znpy2017025).

\section{Compliance with Ethical Standards}

All procedures performed in the study were in accordance with the ethical standards of the Wuhan Central Hospital Ethics Committee and with the 1964 Helsinki Declaration and its later amendments or comparable ethical standards.

Conflict of Interest The authors declare that they have no conflict of interest. Informed Consent Informed consent was obtained from all the participants of the study and was blinded. Declaration The manuscript has not been published previously and is not under simultaneous review elsewhere.

\section{References}

Adam, E. K., Quinn, M. E., Tavernier, R., McQuillan, M. T., Dahlke, K. A., \& Gilbert, K. E. (2017). Diurnal cortisol slopes and mental and physical health outcomes: A systematic review and meta-analysis. Psychoneuroendocrinology, 83, 25-41.

Ai, T., Yang, Z., Hou, H., Zhan, C., Chen, C., Lv, W., et al. (2020). Correlation of chest $\mathrm{CT}$ and RT-PCR testing in coronavirus disease 2019 (COVID-19) in China: A report of 1014 cases. Radiology, 296, E32-E40.

Berlinberg, E. J., Gonzales, J. A., Doan, T., \& Acharya, N. R. (2019). Association between noninfectious uveitis and psychological stress. JAMA Ophthalmology, 137, 199-205.

Bogoch, I. I., Watts, A., Thomas-Bachli, A., Huber, C., Kraemer, M. U. G., and Khan, K. (2020). Potential for global spread of a novel coronavirus from China. Journal of Travel Medicine, 27, taaa011.

Chan, J. F., Yuan, S., Kok, K. H., To, K. K., Chu, H., Yang, J., et al. (2020). A familial cluster of pneumonia associated with the 2019 
novel coronavirus indicating person-to-person transmission: A study of a family cluster. Lancet (London, England), 395, 514-523.

Chen, Q., Liang, M., Li, Y., Guo, J., Fei, D., Wang, L., et al. (2020). Mental health care for medical staff in China during the COVID-19 outbreak. The lancet. Psychiatry, 7, e15-e16.

Duan, L., \& Zhu, G. (2020). Psychological interventions for people affected by the COVID-19 epidemic. The lancet. Psychiatry, 7, 300 302.

Feinstein, A., Owen, J., \& Blair, N. (2002). A hazardous profession: War, journalists, and psychopathology. The American Journal of Psychiatry, 159, 1570-1575.

Fischer, M., Coogan, A. N., Faltraco, F., \& Thome, J. (2020). COVID-19 paranoia in a patient suffering from schizophrenic psychosis - a case report. Psychiatry Research, 288, 113001.

Garrouste-Orgeas, M., Flahault, C., Vinatier, I., Rigaud, J. P., ThieulotRolin, N., Mercier, E., et al. (2019). Effect of an ICU diary on posttraumatic stress disorder symptoms among patients receiving mechanical ventilation: A randomized clinical trial. Jama, 322, 229-239.

Gonzalez-Sanguino, C., Ausin, B., Castellanos, M. A., Saiz, J., LopezGomez, A., Ugidos, C., \& Munoz, M. (2020). Mental health consequences during the initial stage of the 2020 coronavirus pandemic (COVID-19) in Spain. Brain, Behavior, and Immunity, 87, 172176

Heymann, D. L., \& Shindo, N. (2020). COVID-19: What is next for public health? Lancet (London, England), 395, 542-545.

Holshue, M. L., DeBolt, C., Lindquist, S., Lofy, K. H., Wiesman, J., Bruce, H., Spitters, C., Ericson, K., Wilkerson, S., Tural, A., Diaz, G., Cohn, A., Fox, L., Patel, A., Gerber, S. I., Kim, L., Tong, S., Lu, X., Lindstrom, S., Pallansch, M. A., Weldon, W. C., Biggs, H. M., Uyeki, T. M., Pillai, S. K., \& Washington State 2019-nCoV Case Investigation Team. (2020). First case of 2019 novel coronavirus in the United States. The New England Journal of Medicine, 382, 929 936

Huang, C., Wang, Y., Li, X., Ren, L., Zhao, J., Hu, Y., et al. (2020). Clinical features of patients infected with 2019 novel coronavirus in Wuhan, China. Lancet (London, England), 395, 497-506.

Kang, L., Li, Y., Hu, S., Chen, M., Yang, C., Yang, B. X., Wang, Y., Hu, J., Lai, J., Ma, X., Chen, J., Guan, L., Wang, G., Ma, H., \& Liu, Z. (2020a). The mental health of medical workers in Wuhan, China dealing with the 2019 novel coronavirus. Lancet Psychiatry, 7, e14.

Kang, L., Ma, S., Chen, M., Yang, J., Wang, Y., Li, R., Yao, L., Bai, H., Cai, Z., Xiang Yang, B., Hu, S., Zhang, K., Wang, G., Ma, C., \& Liu, Z. (2020b). Impact on mental health and perceptions of psychological care among medical and nursing staff in Wuhan during the 2019 novel coronavirus disease outbreak: A cross-sectional study. Brain, Behavior, and Immunity, 87, 11-17.

Katsarou, A., Panagiotakos, D., Zafeiropoulou, A., Vryonis, M., Skoularigis, I., Tryposkiadis, F., \& Papageorgiou, C. (2012). Validation of a Greek version of PSS-14; a global measure of perceived stress. Central European Journal of Public Health, 20, 104 109

Li, Q., Guan, X., Wu, P., Wang, X., Zhou, L., Tong, Y., Ren, R., Leung, K. S. M., Lau, E. H. Y., Wong, J. Y., Xing, X., Xiang, N., Wu, Y., Li, C., Chen, Q., Li, D., Liu, T., Zhao, J., Liu, M., Tu, W., Chen, C., Jin, L., Yang, R., Wang, Q., Zhou, S., Wang, R., Liu, H., Luo, Y., Liu, Y., Shao, G., Li, H., Tao, Z., Yang, Y., Deng, Z., Liu, B., Ma, Z., Zhang, Y., Shi, G., Lam, T. T. Y., Wu, J. T., Gao, G. F., Cowling, B. J., Yang, B., Leung, G. M., \& Feng, Z. (2020). Early transmission dynamics in Wuhan, China, of novel coronavirusinfected pneumonia. The New England Journal of Medicine, 382, $1199-1207$.

Mahase, E. (2020). Coronavirus covid-19 has killed more people than SARS and MERS combined, despite lower case fatality rate. BMJ (Clinical Research ed.), 368, m641.
Mbala-Kingebeni, P., Villabona-Arenas, C. J., Vidal, N., Likofata, J., Nsio-Mbeta, J., Makiala-Mandanda, S., Mukadi, D., Mukadi, P., Kumakamba, C., Djokolo, B., Ayouba, A., Delaporte, E., Peeters, M., Muyembe Tamfum, J. J., \& Ahuka-Mundeke, S. (2019). Rapid confirmation of the Zaire Ebola virus in the outbreak of the Equateur Province in the Democratic Republic of Congo: Implications for public health interventions. Clinical Infectious Diseases : An Official Publication of the Infectious Diseases Society of America, 68, 330-333.

Metsky, H. C., Matranga, C. B., Wohl, S., Schaffner, S. F., Freije, C. A., Winnicki, S. M., West, K., Qu, J., Baniecki, M. L., Gladden-Young, A., Lin, A. E., Tomkins-Tinch, C. H., Ye, S. H., Park, D. J., Luo, C. Y., Barnes, K. G., Shah, R. R., Chak, B., Barbosa-Lima, G., Delatorre, E., Vieira, Y. R., Paul, L. M., Tan, A. L., Barcellona, C. M., Porcelli, M. C., Vasquez, C., Cannons, A. C., Cone, M. R., Hogan, K. N., Kopp, E. W., Anzinger, J. J., Garcia, K. F., Parham, L. A., Ramírez, R. M. G., Montoya, M. C. M., Rojas, D. P., Brown, C. M., Hennigan, S., Sabina, B., Scotland, S., Gangavarapu, K., Grubaugh, N. D., Oliveira, G., Robles-Sikisaka, R., Rambaut, A., Gehrke, L., Smole, S., Halloran, M. E., Villar, L., Mattar, S., Lorenzana, I., Cerbino-Neto, J., Valim, C., Degrave, W., Bozza, P. T., Gnirke, A., Andersen, K. G., Isern, S., Michael, S. F., Bozza, F. A., Souza, T. M. L., Bosch, I., Yozwiak, N. L., MacInnis, B. L., \& Sabeti, P. C. (2017). Zika virus evolution and spread in the Americas. Nature, 546, 411-415.

Nishiura, H., Jung, S. M., Linton, N. M., Kinoshita, R., Yang, Y., Hayashi, K., et al. (2020). The extent of transmission of novel coronavirus in Wuhan, China, 2020. Journal of Clinical Medicine, 9, 330.

Pariante, C. M. (2016). Neuroscience, mental health and the immune system: Overcoming the brain-mind-body trichotomy. Epidemiology and Psychiatric Sciences, 25, 101-105.

Park, S. C., \& Jeen, Y. T. (2016). The mental health state of quiescent inflammatory bowel disease patients. Gut and Liver, 10, 330-331.

Pfefferbaum, B., Schonfeld, D., Flynn, B. W., Norwood, A. E., Dodgen, D., Kaul, R. E., Donato, D., Stone, B., Brown, L. M., Reissman, D. B., Jacobs, G. A., Hobfoll, S. E., Jones, R. T., Herrmann, J., Ursano, R. J., \& Ruzek, J. I. (2012). The H1N1 crisis: A case study of the integration of mental and behavioral health in public health crises. Disaster Medicine and Public Health Preparedness, 6, 67-71.

Qiu, W., Chu, C., Mao, A., \& Wu, J. (2018). The impacts on health, society, and economy of SARS and H7N9 outbreaks in China: A case comparison study. Journal of Environmental and Public Health, 2018, 2710185.

Riou, J., \& Althaus, C.L. (2020). Pattern of early human-to-human transmission of Wuhan 2019 novel coronavirus (2019-nCoV), December 2019 to January 2020. Euro surveillance : Bulletin Europeen Sur les maladies transmissibles. European Communicable Disease Bulletin, 25,2000058

Stefana, A., Youngstrom, E. A., Chen, J., Hinshaw, S., Maxwell, V., Michalak, E., \& Vieta, E. (2020). The COVID-19 pandemic is a crisis and opportunity for bipolar disorder. Bipolar Disorders, 22, 641-643.

The, L. (2020). COVID-19: Fighting panic with information. Lancet, 395, 537. ((London, England)).

Wang, C., Pan, R., Wan, X., Tan, Y., Xu, L., Ho, C. S., \& Ho, R. C. (2020). Immediate psychological responses and associated factors during the initial stage of the 2019 coronavirus disease (COVID-19) epidemic among the general population in China. International Journal of Environmental Research and Public Health, 17, 1729.

Wang, D., Hu, B., Hu, C., Zhu, F., Liu, X., Zhang, J., et al. (2020). Clinical characteristics of 138 hospitalized patients with 2019 novel coronavirus-infected pneumonia in Wuhan, China. Journal of the American Medical Association, 323, 1061-1069.

Wu, J. T., Leung, K., \& Leung, G. M. (2020). Nowcasting and forecasting the potential domestic and international spread of the 2019- 
nCoV outbreak originating in Wuhan, China: A modelling study. Lancet (London, England), 395, 689-697.

Xu, Z., Shi, L., Wang, Y., Zhang, J., Huang, L., Zhang, C., et al. (2020). Pathological findings of COVID-19 associated with acute respiratory distress syndrome. The Lancet. Respiratory Medicine, 8, 420422.

Yang, Y., Shang, W., \& Rao, X. (2020). Facing the COVID-19 outbreak: What should we know and what could we do? Journal of Medical Virology, 92, 536-537.
Zung, W. W., \& Wonnacott, T. H. (1970). Treatment prediction in depression using a self-rating scale. Biological Psychiatry, 2, 321-329.

Publisher's Note Springer Nature remains neutral with regard to jurisdictional claims in published maps and institutional affiliations. 